\title{
Indicadores de aptidão física de escolares da região centro-oeste da cidade de São Paulo
}

\author{
Indicators of physical fitness in school children from the midwest \\ region of São Paulo City
}

\author{
Carla Nascimento Luguetti ${ }^{1,2}$ \\ Alessandro H. Nicolai Ré ${ }^{1,3}$ \\ Maria Tereza Silveira Böhme
}

1 Universidade de São Paulo. Escola de Educação Física e Esporte. São Paulo, SP. Brasil

2 Universidade Santa Cecília, Faculdade de Educação Física e Esporte. São Paulo, SP. Brasil

3 Universidade de São Paulo. Escola de Artes, Ciências e Humanidades. São Paulo, SP. Brasil

Recebido em 23/05/09 Revisado em 12/08/09 Aprovado em 13/05/10
Resumo - Os objetivos deste estudo foram: a) mensurar indicadores de aptidão física em crianças e adolescentes, de acordo com a idade cronológica e o sexo; b) classificar seu desempenho por meio das tabelas normativas do PROESP-BR. Participaram do estudo 3145 escolares selecionados aleatoriamente (1590 meninos e 1555 meninas), com idades entre 7 e 16 anos, que realizaram os seguintes testes: distância percorrida em 9 minutos de corrida, salto horizontal, arremesso de medicine-ball e flexão abdominal em 1 minuto. Foram verificadas diferenças estatisticamente significantes entre os sexos em todas as idades cronológicas, bem como nas comparações entre os jovens do sexo masculino de diferentes idades cronológicas. No sexo feminino, não houve diferenças significantes no fator idade cronológica, existindo, ainda, uma tendência de estabilização do desempenho a partir dos 11/12 anos de idade. Classificando-se os jovens nas tabelas normativas do PROESP-BR, observaram-se valores elevados, em geral, superiores a 50\%, para a classificação "ruim" (abaixo do percentil 40) em todos os testes para ambos os sexos, principalmente, no feminino; essa mesma tendência foi observada na comparação com outros estudos brasileiros. Considerando os valores médios obtidos, concluiu-se que os jovens, principalmente, do sexo feminino, apresentam um baixo nível de aptidão física, fato que justifica a necessidade de programas de promoção de saúde na região centro oeste de São Paulo, especialmente, voltados à prática de atividades físicas.

Palavras-chave: Aptidão física; Saúde escolar; Atividade motora.

Abstract - The objectives of this study were: a) to evaluate indicators of physical fitness in children and adolescents according to chronological age and gender; b) to classify their performance using the PROESP-BR normative tables. A total of 3145 randomly selected school children (1590 boys and 1555 girls), ranging in age from 7 to 16 years, were submitted to the following tests: distance covered in a 9-minute run, standing long jump test, medicine-ball throw, and 60-s situp test to measure abdominal muscle endurance. Significant differences were observed between genders at all chronological ages and also between boys of different chronological ages. In girls, no significant differences were observed between chronological ages, with performance tending to stabilize at 11/12 years of age. Classification of the participants according to the PROESP-BR normative tables showed that more than $50 \%$ of all subjects were below the $40^{\text {th }}$ percentile in all tests, especially girls. The same tendency was observed when compared to other Brazilian studies. Considering mean values, it can be concluded that the school children studied, especially girls, presented a low level of physical fitness, thus indicating the need for health promotion programs in the midwest region of São Paulo City addressing physical activity.

Key words: Physical fitness; School Health; Motor Activity. 


\section{INTRODUÇÃO}

A aptidão física refere-se à capacidade do indivíduo apresentar um desempenho físico adequado em suas atividades diárias; pode ser relacionada com a saúde e aptidão motora, ou destrezas e habilidades esportivas ${ }^{1}$. Índices satisfatórios dos aspectos de aptidão física relacionados à saúde oferecem alguma proteção ao aparecimento e desenvolvimento de distúrbios orgânicos. De tal forma que, melhores índices cardiorrespiratórios, de força/resistência muscular, flexibilidade, assim como níveis adequados de gordura corporal, estão diretamente associados com um menor risco para o desenvolvimento de doenças degenerativas. Assim, a aquisição de hábitos positivos para a prática de atividade física na infância pode repercutir de forma positiva no estado de aptidão física e saúde durante a vida adulta ${ }^{2,3}$.

Essa preocupação com a melhora da qualidade de vida das pessoas torna-se uma questão de grande importância social, que leva ao surgimento de pesquisas com o propósito de investigar a aptidão física de crianças e adolescentes, assim como estudos populacionais, que meçam a eficiência cardiovascularrespiratória e neuromuscular de crianças e adolescentes na perspectiva de saúde e, consequentemente, estabelecer comparações intra e interpopulações, permitindo ações intervencionistas.

Diversos estudos apontam para um declínio nos índices de aptidão física de crianças e adolescentes, evidenciado em estudos de caráter longitudinal ${ }^{4}$, transversal ${ }^{5,6}$ e misto ${ }^{7}$. Contudo, observa-se uma carência de dados atualizados, sobretudo, da realidade de São Paulo, cujas características urbanas, possivelmente, limitem a prática espontânea de atividades físicas, repercutindo nos índices de aptidão física.

Verificar os aspectos da aptidão física relacionados à saúde de jovens poderá contribuir de forma decisiva na tentativa de promoção da saúde coletiva. E ainda, existe a necessidade de parâmetros referenciais com escolares brasileiros, pois dados normativos de escolares de países desenvolvidos não equivalem aos de países em desenvolvimento. Resumidamente, as diferenças em fatores sociais, econômicos e culturais justificam a realização de estudos regionais, ao invés da utilização de dados internacionais.

Nesse sentido, em 2003, foi instituído o Projeto Esporte Brasil (PROESP-BR), que tem por objetivo delinear fatores de aptidão motora em crianças e adolescentes na faixa etária entre 7 a 16 anos, tendo em vista constituir indicadores que forneçam subsídios adicionais para a elaboração de políticas de educação física e esportes para crianças e jovens no Brasil ${ }^{8}$.

Portanto, os objetivos deste estudo, por meio de um delineamento transversal, foram: a) mensurar indicadores de aptidão física em crianças e adolescentes, de acordo com a idade cronológica e o sexo; b) classificar seu desempenho por meio das tabelas normativas do PROESP-BR.

\section{PROCEDIMENTOS METODOLÓGICOS}

Participaram do estudo, 3145 escolares da rede pública da cidade de São Paulo, na faixa etária de 7 a 16 anos, sendo 1590 do sexo masculino e 1555 do sexo feminino. Para o processo de amostragem, utilizou-se como unidade amostral as escolas estaduais pertencentes à Diretoria de Ensino da região centro-oeste da cidade de São Paulo, no ano de 2003 (n=14) e selecionadas aleatoriamente sete escolas.

O critério de inclusão para participar do estudo era de que o aluno estivesse regularmente matriculado no ensino fundamental, no ano de 2003, com idade entre 7 e 16 anos. Não foram avaliados os escolares que: a) possuíam contraindicação médica para a realização de exercícios físicos; b) não obtiveram autorização dos pais ou responsável para realizar as avaliações; c) se recusaram a participar das avaliações; d) não compareceram à aula no dia das avaliações.

Os pais ou responsáveis assinaram um termo de consentimento para que os jovens pudessem participar da pesquisa, de acordo com a resolução 196/96 do Conselho Nacional de Saúde ${ }^{9}$ para pesquisas em seres humanos. $\mathrm{O}$ presente estudo faz parte do PROESP-BR que foi aprovado pelo Comitê de Ética em Pesquisa da UFRGS no processo CEP-UFRGS 2007719.

Para a avaliação dos indicadores de aptidão física, foram utilizados testes indicadores da resistência cardiorrespiratória (distância percorrida em 9 minutos), da força explosiva de membros inferiores (salto horizontal), da força explosiva de membros superiores (arremesso de medicine-ball de $2 \mathrm{Kg}$ ) e da força/resistência abdominal (número máximo de repetições em 1 minuto), de acordo com as padronizações sugeridas ${ }^{8,10}$.

Em todas as medidas, os avaliados realizaram duas tentativas, anotando-se o maior valor, exceto no teste de corrida de 9 minutos, realizado uma única vez. Todos os testes possuem um bom nível de autenticidade científica e foram aplicados por cinco professores de Educação Física, devidamente capacitados, sendo que cada um deles ficou respon- 
sável pela coleta de uma medida e/ou teste, a fim de evitar a variabilidade interavaliador e garantir a fidedignidade dos dados. O cálculo das idades cronológicas foi feito considerando-se a data de nascimento e o dia dos testes.

Como critério de avaliação do nível de aptidão física dos participantes, foram utilizadas tabelas normativas apresentadas para cada idade e sexo, disponíveis no site do PROESP ${ }^{8}$. O PROESP-BR adota um sistema referenciado em normas, tendo como referência uma amostra de 100 mil crianças e jovens brasileiros, estratificados por idade e sexo. A aptidão é classificada em seis categorias: a) muito fraco: valores inferiores ao percentil 20 (norma utilizada como critério referenciado ao risco à saúde); b) fraco: valores entre o percentil 20 e 40; c) razoável: valores entre o percentil 40 e 60 ; d) bom: valores entre o percentil 60 e 80 ; e) muito bom: valores entre o percentil 80 e 98 ; f) excelente: acima do percentil 98. Para a análise dos dados, agrupamos: a) muito fraco e fraco, classificando como "ruim" (até o percentil 40); b) razoável e bom, classificando como "normal" (entre os percentis 40 e 80); c) muito bom e excelente, classificando como "bom" (acima do percentil 80).

Para a análise estatística, primeiramente, foi confirmada a existência de distribuição normal dos dados e de igualdade de variâncias (homocedasticidade), por meio dos testes de Kolmogorov-Smirnov $e$ de Levene, respectivamente, de acordo com as recomendações da literatura ${ }^{11,12}$.

Com os pressupostos necessários para a utilização da estatística paramétrica devidamente atendidos, os resultados foram comparados, utilizando-se a Análise de Variância (ANOVA) a 2 fatores [2 (sexos) x 10 (faixas etárias)]. Os contrastes posteriores (post-hoc), quando necessários, foram feitos por meio do teste Tukey. No entanto, foram consideradas apenas as eventuais diferenças entre idades adjacentes.

Classificou-se o desempenho dos testes de aptidão física para cada sexo e idade de acordo com as tabelas normativas do PROESP-BR. Para comparar o desempenho com os resultados de estudos realizados em outras regiões do Brasil, utilizaram-se estudos até 1982 que utilizaram a mesma bateria de testes do presente estudo, e que apresentaram dados normativos. Para a análise estatística, foi utilizado o programa estatístico SPSS for Windows 13.0. Em todas as comparações foi utilizado o nível de significância de 5\%.

\section{RESULTADOS}

Foram verificadas diferenças estatisticamente significantes para os fatores sexo e idade, em todas as medidas realizadas. Os valores médios de cada variável são descritos na Tabela 1.

Em relação ao sexo, observaram-se diferenças estatisticamente significantes em todas as variáveis e idades, exceto aos 7 anos para os testes de corrida de 9 minutos e de força abdominal. Em termos percentuais, as diferenças entre os sexos oscilaram entre 7 e $10 \%$ aos 7 anos e entre 23 e $40 \%$ aos 16 anos, em favor dos meninos.

Nas figuras 1 a 4, são apresentadas distribuições percentuais para as classificações da aptidão física em cada idade e sexo.

Tabela 1. Resultados médios (desvio padrão) das variáveis mensuradas

\begin{tabular}{|c|c|c|c|c|c|c|c|c|}
\hline \multirow[t]{2}{*}{$\begin{array}{l}\text { Idade } \\
\text { (anos) }\end{array}$} & \multicolumn{2}{|c|}{ Corrida de 9 minutos $(\mathrm{m})$} & \multicolumn{2}{|c|}{ Salto horizontal $(\mathrm{cm})$} & \multicolumn{2}{|c|}{$\begin{array}{l}\text { Arremesso de medicine-ball } \\
\qquad(\mathrm{cm})\end{array}$} & \multicolumn{2}{|c|}{ Abdominal (repetições) } \\
\hline & Masc & Fem & Masc & Fem & Masc & Fem & Masc & Fem \\
\hline 7 & $1027(200)$ & $1040(181)$ & $112(17)$ & $99(15)^{+}$ & $158(29)$ & $144(23)^{+}$ & $20(10)$ & $18(10)$ \\
\hline 8 & $1156(200) *$ & $1076(159)^{+}$ & $122(18)$ & $108(18)^{*+}$ & $180(36)$ & $163(27)^{*+}$ & $23(10)$ & $18(9)^{+}$ \\
\hline 9 & 1171 (216) & $1109(175)^{+}$ & $131(18)$ & $116(18)^{+}$ & 201 (35) & $189(36)^{+}$ & $26(9)$ & $20(9)^{+}$ \\
\hline 10 & $1260(207) *$ & $1171(167)^{+}$ & $136(20)$ & $123(19)^{+}$ & $232(46) *$ & $216(42)^{*+}$ & $28(10)$ & $24(9)^{*+}$ \\
\hline 11 & $1284(202)$ & $1138(156)^{+}$ & $136(21)$ & $124(20)^{+}$ & $266(47) *$ & $250(47)^{*+}$ & $30(9)$ & $23(9)^{+}$ \\
\hline 12 & $1353(206)$ * & $1131(147)^{+}$ & $144(25)$ * & $123(19)^{+}$ & $301(49) *$ & $281(49)^{*+}$ & $32(8)$ & $25(9)^{+}$ \\
\hline 13 & 1400 (194) & $1170(149)^{+}$ & $160(23)$ * & $125(23)^{+}$ & $374(71) *$ & $314(48)^{*+}$ & $35(9)$ & $27(8)^{+}$ \\
\hline 14 & $1435(207)$ & $1149(170)^{+}$ & $171(26)$ * & $128(19)^{+}$ & $418(76) *$ & $321(43)^{+}$ & $38(9)$ & $25(8)^{+}$ \\
\hline 15 & $1526(218) *$ & $1151(149)^{+}$ & $180(28)$ * & $131(20)^{+}$ & $467(80) *$ & $323(42)^{+}$ & $38(9)$ & $28(7)^{+}$ \\
\hline 16 & 1581 (199) & $1206(161)^{+}$ & $187(25)$ & $133(20)^{+}$ & $487(80)$ & $331(50)^{+}$ & $38(9)$ & $25(7)^{+}$ \\
\hline
\end{tabular}

* diferença em relação à idade anterior, considerando-se o mesmo sexo ( $p<0,05$, post-hoc de Tukey).

${ }^{+}$diferença entre os sexos, considerando-se a mesma idade $(p<0,05)$ 


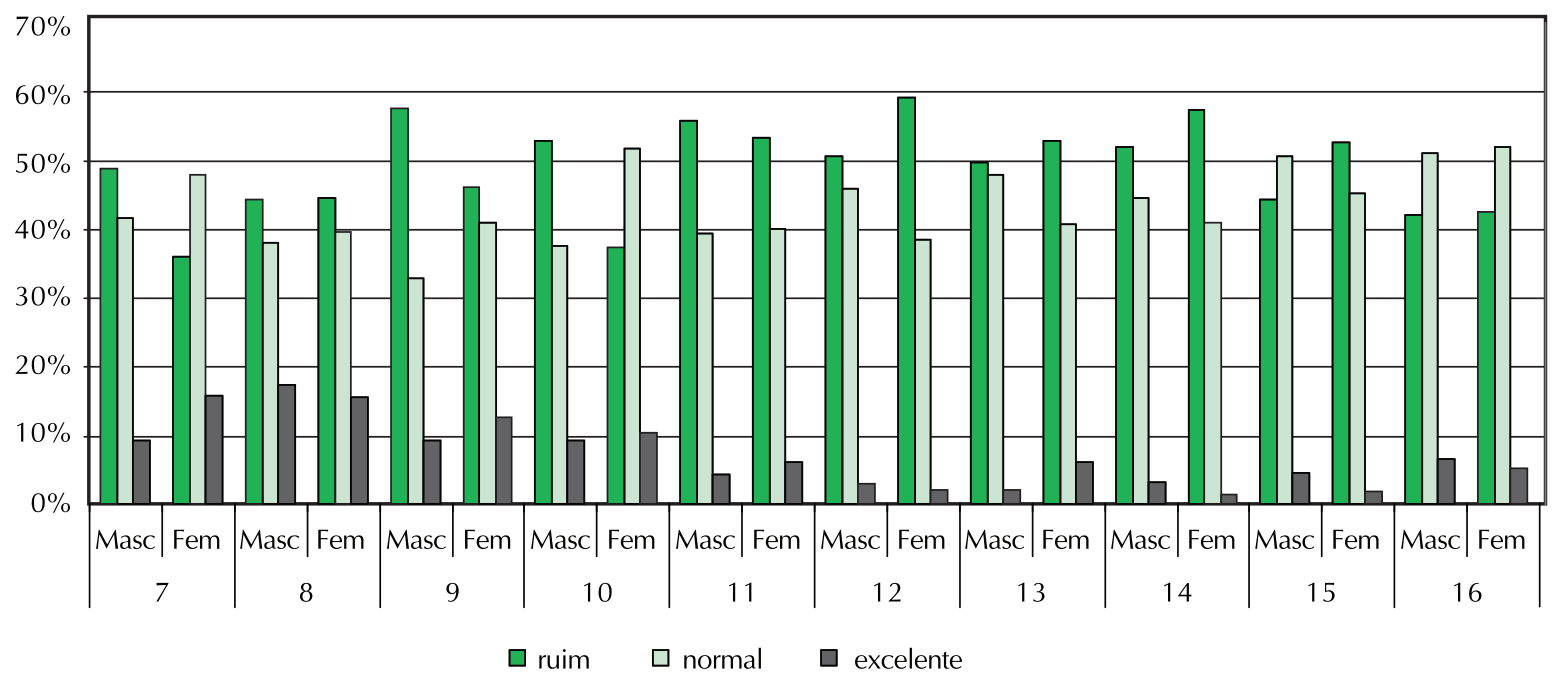

Figura 1. Percentual das classificações do teste de corrida de 9 minutos, para cada idade e sexo.

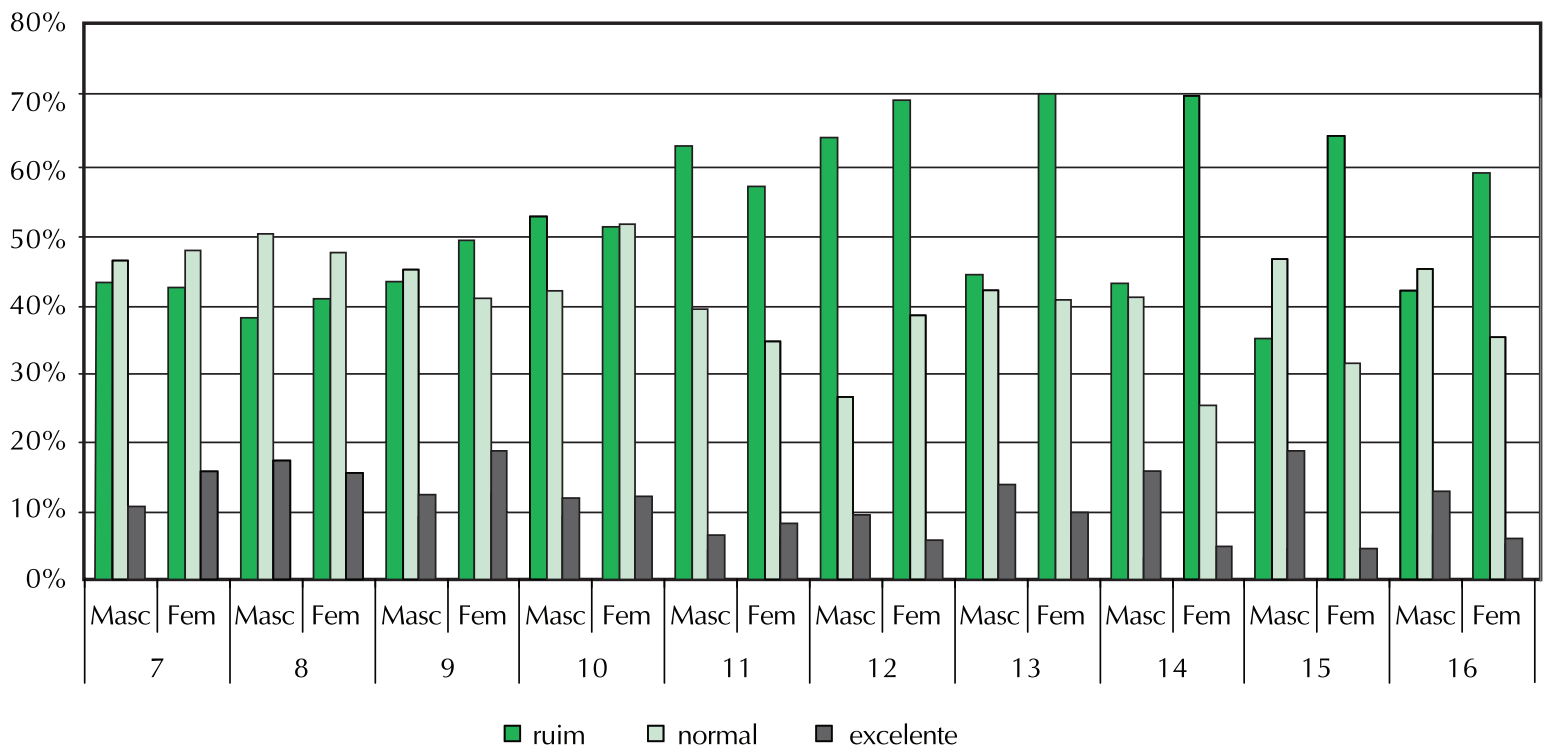

Figura 2. Percentual das classificações do teste de salto horizontal, para cada idade e sexo.

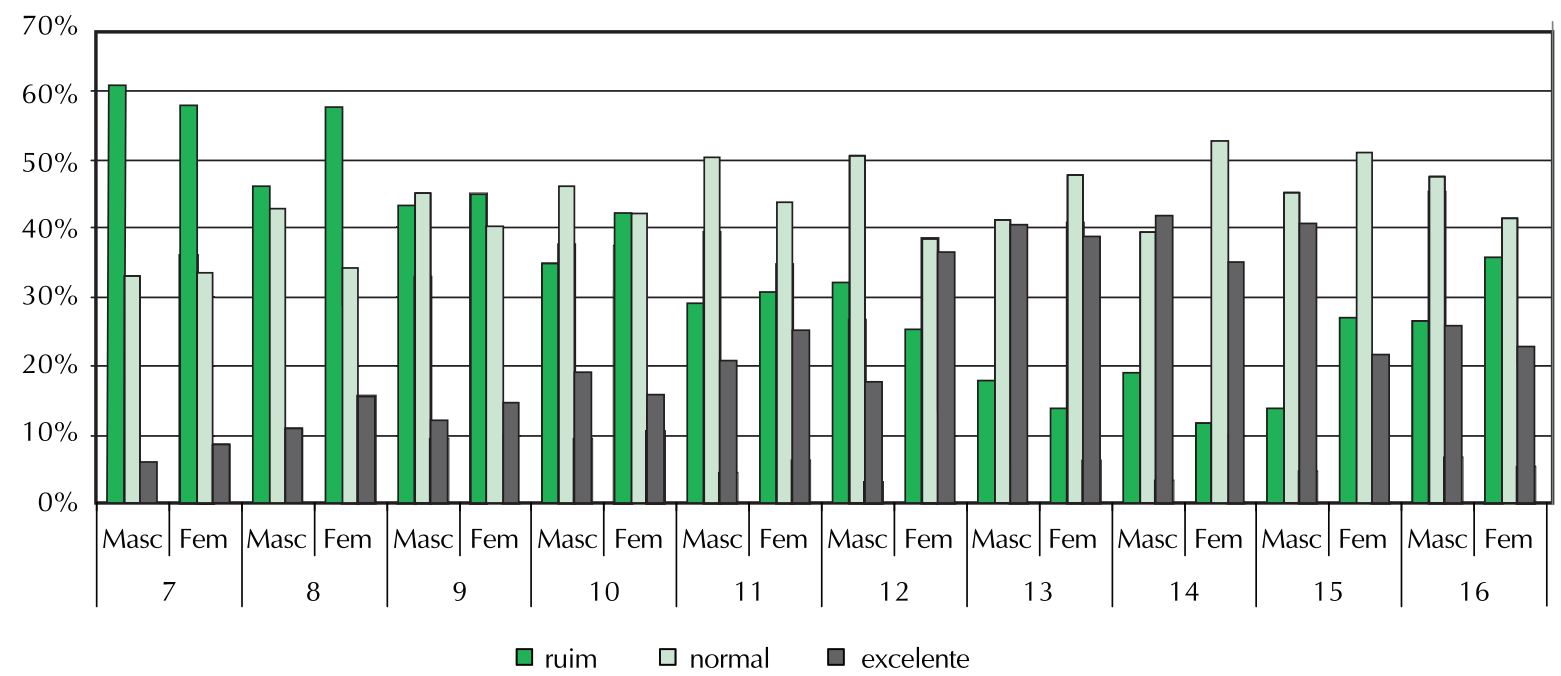

Figura 3. Percentual das classificações do teste de arremesso de medicine-ball, para cada idade e sexo. 


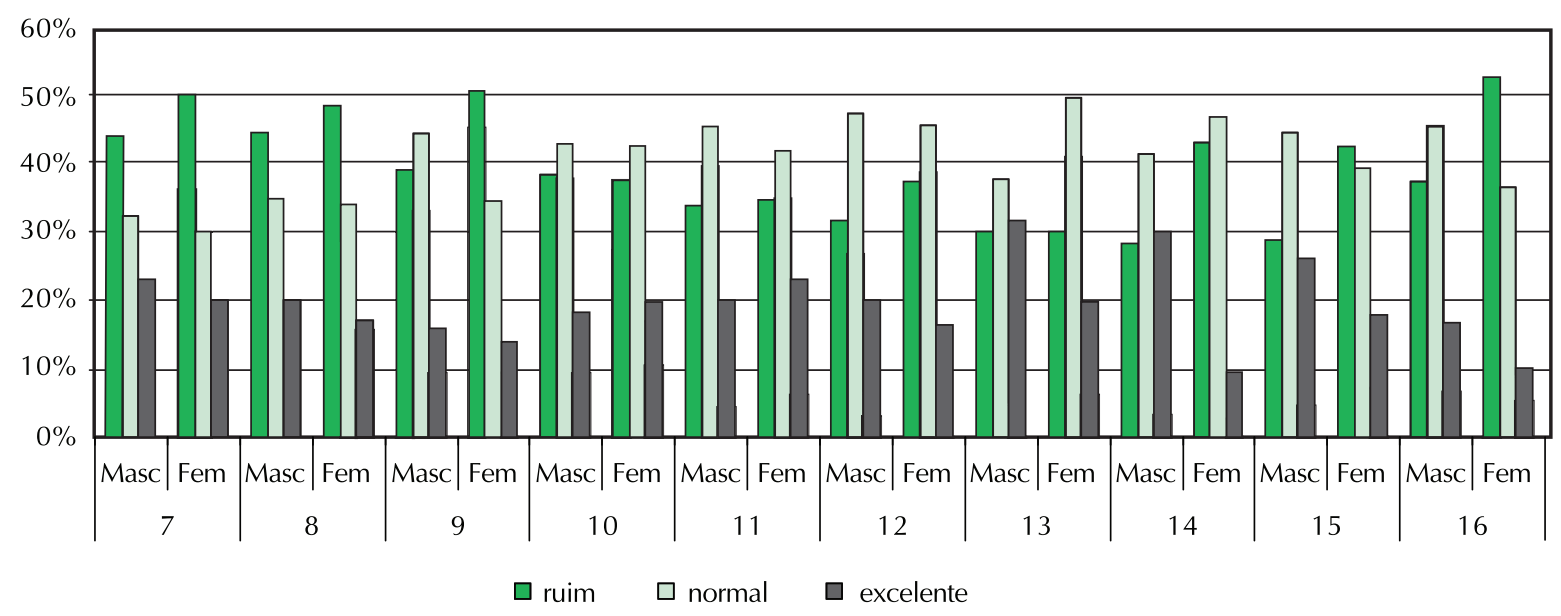

Figura 4. Percentual das classificações do teste de abdominal, para cada idade e sexo.

Para os testes de arremesso de corrida de 9 minutos e salto horizontal, observaram-se elevados valores da classificação "ruim", assim como baixos valores para a classificação "excelente". Verificaramse valores médios de $48 \%$ dos meninos e $50 \%$ das meninas classificados como "ruim", no teste de corrida de 9 minutos, assim como $46 \%$ dos meninos e $57 \%$ das meninas no teste de salto horizontal.

No teste de arremesso de medicine-ball, os valores médios para o sexo masculino foram de $32 \%$, 44\% e 23\% para as classificações "ruim", "normal" e "excelente"; para o sexo feminino observou-se uma classificação de 35\%, 43\% e 23\%, para "ruim", "normal" e "excelente", respectivamente. Observou-se que, conforme a idade, o percentual da classificação "excelente" aumentou em ambos os sexos. Os percentuais de classificação no teste de flexão abdominal, em média, foram de 36\% "ruim", $42 \%$ "normal" e 22\% "excelente", nos meninos, e 43\% "ruim", 40\% "normal" e 17\% "excelente" nas meninas.

As meninas mostraram-se diferentes dos meninos nos percentuais de classificação após 13/14 anos para o teste de salto horizontal, arremesso de medicine-ball e flexão abdominal, observando-se uma maior frequência na classificação "ruim" em comparação com os meninos.

\section{DISCUSSÃO}

A mensuração da aptidão física de crianças e adolescentes, com características sócioculturais relativamente semelhantes, é de fundamental importância para a geração de conhecimento atualizado e específico a determinada população, uma vez que diferenças geográficas, sociais e culturais podem interferir nos valores apresentados ${ }^{13}$. Considerando os resultados obtidos pelos estudantes de escolas públicas situadas na região centro-oeste da cidade de
São Paulo, pode ser afirmado que há a necessidade de programas de intervenção que estimulem uma melhoria nos índices de aptidão física.

Nos testes realizados, foram observadas diferenças estatisticamente significantes entre os sexos em todas as idades, com as únicas exceções aos 7 anos, nos testes de corrida de 9 minutos e de flexão abdominal de 1 minuto. Até, aproximadamente, os 10-11 anos de idade, não existem diferenças importantes na composição corporal que justifiquem a superioridade do sexo masculino em medidas de aptidão física ${ }^{14,15}$. Desse modo, as diferenças encontradas nessa faixa etária podem indicar um menor envolvimento com a prática regular de atividades físicas no sexo feminino, uma vez que existe uma forte relação entre o nível de aptidão física e a prática de atividades físicas de crianças e adolescentes $^{16-19}$.

A estabilização de desempenho no sexo feminino após os 11 anos foi verificada em outros estudos brasileiros ${ }^{5,20-24}$. A partir dos 10-11 anos de idade, é cada vez maior o número de meninas que já apresentaram a menarca ${ }^{25,26}$, e este fato tem importante interferência nos índices de aptidão física feminina. A menarca é diretamente associada ao surgimento de características sexuais do corpo feminino adulto, destacando-se um aumento no percentual de gordura corporal. Desse modo, após a menarca, pode ocorrer alguma desvantagem no desempenho motor, fato que pode ser especulado como uma possível causa da estabilização no sexo feminino após essa faixa etária.

Todavia, de modo geral, quanto menos ativa for a menina em idades anteriores à menarca, maior será a probabilidade de sedentarismo após a menarca ${ }^{27,28}$. Sendo assim, uma das estratégias para o aumento nos índices de aptidão física da população feminina, incluindo crianças e adultas, 
é o envolvimento com a atividade física desde os primeiros anos de vida, evidentemente, de modo adequado às diferentes etapas do desenvolvimento humano. Okano et al. ${ }^{29}$ citam que existe um tratamento diferenciado por parte dos adultos para com os meninos e as meninas durante a infância, sendo que as meninas tendem a brincadeiras mais sedentárias enquanto os garotos são motivados a praticarem atividades dinâmicas que exigem, muitas vezes, a prática moderada e intensa de atividades físicas. Provavelmente, devido a fatores culturais, as meninas têm menor acesso ao movimento em comparação aos meninos, e esse aspecto pode estar relacionado com as diferenças encontradas entre ambos os sexos ${ }^{30}$.

Em contrapartida, diferenças sócioeconômicas podem não explicar baixos valores de aptidão física; Dumith et al. ${ }^{13}$ avaliaram crianças brasileiras de escolas públicas e privadas, verificando que os desempenhos dos alunos não apresentaram grandes diferenças. Concluíram que a aptidão física de escolares depende, relativamente, pouco do nível socioeconômico. Os autores acreditam que os professores de Educação Física têm um papel fundamental no trabalho de aprimoramento desses componentes da aptidão física em suas aulas.

No sexo masculino, apesar da superioridade encontrada em relação ao feminino, os índices de aptidão física encontrados também podem ser considerados ruins, uma vez que, em relação à classificação dos jovens nas tabelas normativas propostas pelo PROESP-BR, o nível de aptidão pode ser considerado fraco. No teste de corrida de $9 \mathrm{mi}$ nutos, os valores médios para a classificação "ruim" foram altos em ambos os sexos (48\% das meninas e $50 \%$ dos meninos), assim como os valores de "excelentes" mostraram-se baixos (8\% das meninas e $7 \%$ dos meninos). Esses resultados revelam uma baixa aptidão cardiorrespiratória em ambos os sexos avaliados, tendo como critério o desempenho de jovens brasileiros. Tais dados tornam-se preocupantes, uma vez que a aptidão cardiorrespiratória pode ser considerada um dos principais componentes da aptidão física, diretamente associada com a saúde e com a prática de atividade física.

Os valores apresentados no teste de corrida de 9 minutos e salto horizontal do presente estudo foram inferiores aos de outras pesquisas brasileiras, realizadas com crianças de Londrina ${ }^{20}$, Bahia ${ }^{5,21}$, Minas Gerais ${ }^{22,23}$ e São Caetano do Sul24. Apesar das crianças serem de locais diferentes à amostra do presente estudo, pode-se sugerir a existência de uma tendência secular negativa dessa variável, ou seja, dos anos 80 e 90 até a presente data, o desempenho das crianças e jovens em atividades aeróbias vem piorando. Utilizando dados longitudinais ou mistos, outros pesquisadores constataram essa tendência no Brasil $1^{5}$ e em outros países ${ }^{4,6,7}$.

\section{CONCLUSÃO}

Em todas as faixas etárias, houve uma superioridade do sexo masculino nas variáveis de aptidão física analisadas, o que sugere uma menor adesão feminina à prática de atividades físicas desde as idades iniciais da presente pesquisa. Todavia, tendo as tabelas normativas do PROESP-BR como critério de referência, em ambos os sexos, foram observados índices elevados para a classificação "ruim" (abaixo do percentil 40). Do mesmo modo, os valores encontrados no presente estudo foram inferiores aos de outras pesquisas de diferentes regiões brasileiras, indicando que os jovens da região centro-oeste da cidade de São Paulo apresentam baixos níveis de aptidão física relacionada à saúde.

As causas relacionadas a esse baixo nível de aptidão física necessitam ser mais bem esclarecidas, especialmente, em grandes centros urbanos como a cidade de São Paulo, cujas características podem estar provocando uma diminuição no envolvimento com a prática regular de atividades físicas, o que resultaria no baixo nível de aptidão física encontrado. Portanto, existe a necessidade de criação de políticas públicas de incentivo à atividade física e de novas pesquisas que visem um maior entendimento das relações entre o estilo de vida dos jovens e seus respectivos índices de aptidão física.

\section{REFERÊNCIAS BIBLIOGRÁFICAS}

1. Böhme MTS. Relações entre aptidão física, esporte e treinamento esportivo. Rev Bras Ciên Mov 2003;11(3):97-104.

2. McGee R, Williams S, Howden-Chapman P, Martin J, Kawachi I. Participation in clubs and groups from childhood to adolescence and its effects on attachment and self-esteem. J Adolesc 2006;29(1):1-17.

3. Nahas, MV. Educação para um estilo de vida ativo nos programas de educação física. In: Nahas, M.V. Atividade física, saúde e qualidade de vida. Londrina: Ed. Midiograf; 2001.

4. Hamlin M.; Ross J.; Hong SW. Health-related fitness trends in 6- to 12-year-old New Zealand children from 1984-85 to 2000. J Sports Sci 2003;21(4):273-82.

5. Dórea VR. Aptidão física e saúde: Um estudo de tendência secular em escolares de 7 a 12 anos de Jequié (BA). [Tese - Doutorado - Programa de Pós graduação da Escola de Educação Física e Esporte]. São Paulo: Universidade de São Paulo; 2004. 
6. Tomkinson GR, Olds TS, Gulbin J. Secular trends in physical performance of Australian children. J Sports Med Phys Fitness 2003;43:47-80.

7. Ferreira JV, Maia JAR, Lopes VP. A mixed-longitudinal study of somatic growth, physical activity, healthrelated physical fitness and motor co-ordination in children from Viseu, Portugal. Rev Port Cien Desp 2003;3(2):155-6.

8. Cenesp-Ufrgs. Projeto Esporte Brasil: Indicadores de saúde e fatores de prestação esportiva em crianças e jovens. [online] 2005; Disponível em: <http://www6. ufrgs.br/esef/proesp-br> [2007 ago 25].

9. Conselho nacional de saúde. Resolução n. ${ }^{\circ}$ 196, de 10 de outubro de 1996. Disponível em http://www. extranet.ceuma.br/downloads_2007/pesquisa/comite_resolucao.pdf.> [2008 mai 9].

10. Safrit MJ. Complete guide to youth fitness testing. Champaign: Human Kinetics; 1995.

11. Hair Jr, Anderson RE, Tatham RL, Black WC. Multivariate data analysis. 5. ed. New Jersey: Prentice Hall; 1998.

12. Neter J, Kutner MH, Nachtsheim CJ, Wasserman W. Applied linear statistical models. Chicago: Irwin; 1996.

13. Dumith SC, Azevedo Jr MR, Rombaldi AJ. Aptidão Física Relacionada à Saúde de Alunos do Ensino Fundamental do Município de Rio Grande, RS, Brasil. Rev Bras Med Esporte 2008;14 (5):454-9.

14. Rowland T W. Developmental exercise physiology. Champaign, IL: Human Kinetics; 1996.

15. Wilmore JH, Costill DL. Physiology of sport and exercise. 3 ed. Champaign: Human Kinetics; 2004.

16. Sollerhed AC, Ejlertsson G. Physical benefits of expanded physical education in primary school: Findings from a 3-year intervention study in Sweden. Scand J Med Sci Sports 2008;18(1):102-7.

17. Haga M. The relationship between physical fitness and motor competence in children. Child Care Health Dev 2008;34(3):329-34.

18. Dunton GF, Schneider M, Cooper DM. An investigation of psychosocial factors related to changes in physical activity and fitness among female adolescents. Psychol Health 2007;22(8):929-44.

19. Rogoff B. A natureza cultural do desenvolvimento humano. Porto Alegre: Artmed; 2005.

20. Guedes DP. Crescimento, composição corporal e desempenho motor em crianças e adolescentes do município de Londrina (PR) [Tese - Doutorado - Programa de Pós-graduação da Escola de Educação Física e Esporte] São Paulo: Universidade de São Paulo;1994.

21. Dórea VR. Aptidão física relacionada a saúde em escolares de Jequié - estado da Bahia. 1990. [Dissertação - Mestrado - Programa de Pós-graduação da Escola de Educação Física e Esporte]. São Paulo: Universidade de São Paulo, 1990.
22. Böhme MTS. Aptidão física e crescimento físico de escolares de 7 a 17 anos de Viçosa- parte I: Resistência aeróbica. Rev Min Educ Fís 1994;2(1):27-41.

23. Böhme MTS. Aptidão física e crescimento físico de escolares de 7 a 17 anos de Viçosa- parte II: Força muscular. Rev Min Educ Fís 1994;2(2) 35-44.

24. Barbanti VJ. A comparative study of selected anthropometric and physical fitness measurements of brazilian and american school children. 1982. [Tese - doutorado] - University of Lowa, 1992.

25. Tavares CHF, Haeffner LSB, Barbieri MA, Bettiol H, Barbieri MR, Souza L. Idade da menarca em escolares de uma comunidade rural do Sudeste do Brasil. Cad Saúde Pública 2000;16(3):709-15.

26. Wellens R, Malina RM, Beunen G, Lefevre J. Age at menarche in Flemish girls: Current status and secular change in the 20th century. Ann Hum Biol 1990;17:145-52

27. Davison KK, Werder JL, Trost SG, Baker BL, Birch LL. Why are early maturing girls less active? Links between pubertal development, psychological well-being, and physical activity among girls at ages 11 and 13 . Soc Sci Med. 2007;64(12):2391-404.

28. Van Den Berg L, Coetzee B, Pienaar AE. The influence of biological maturation on physical and motor performance talent identification determinants of U-14 provincial girl tennis players. J Hum Mov Stud 2006; 50(4):273-90.

29. Okano AH, Altimari LR, Dodero SR, Coelho CF, Almeida PBL, Cyrino ES. Comparação entre o desempenho motor de crianças de diferentes sexos e grupos étnicos. Rev Bras Ciênc Mov 2001;9(3):39-44.

30. Stodden DF, Goodway JD, Langendorfer SJ, Roberton MA, Rudisill ME, Garcia C, Garcia LE. A developmental perspective on the role of motor skill competence in physical activity: An emergent relationship Quest 2008;60(2):290-306.

\author{
Endereço para correspondência \\ Carla Nascimento Luguetti \\ Escola de Educação Física e Esporte - USP \\ Av. Prof. Mello Moraes, 65 \\ 05508-900 - São Paulo - SP - BRASIL \\ E-mail: luguetti@usp.br
}

